\title{
动态共价键化学在酶催化中的应用
}

\author{
张燕 ${ }^{1,2}$, 陈敬华 ${ }^{1,2 *}$
}

1. 江南大学生物工程学院, 糖化学与生物技术教育部重点实验室, 无锡 214122;

2. 江南大学药学院, 无锡 214122

* 联系人, E-mail: chenjinghua@jiangnan.edu.cn

收稿日期：2018-11-02; 接受日期：2018-12-19; 网络版发表日期：2019-02-19

国家自然科学基金(批准号: 21574059)、江苏省自然科学基金(批准号: BK20180625)和轻工技术与工程“双一流”建设学科(批准号: LITE201820)资助

摘要 动态共价键化学是建立在可逆共价反应上的新兴研究方向. 体系中处于动态平衡的化合物库被视为具有 响应性的整体, 环境的改变或外界刺激的加入可诱导其组分的变化, 以获得最优化学结构. 当刺激源为酶催化时, 一方面, 单个可逆反应可实现底物异构体的快速消旋, 在动态动力学拆分的应用中能显著提高酶催化不对称合成 的产率和对映体过量(ee), 具有较高的实际应用价值; 另一方面, 多反应体系的动态组合化学可用于酶催化底物的 笁选, 而多样化的体系形式可有效提高䇻选效率, 获得最佳底物结构, 并提供靶点酶的催化性能、活性位点等信 息, 在生物医药领域有广阔的应用前景.

关键词 动态共价键化学, 酶催化, 动态动力学拆分, 动态组合化学

动态共价键化学是从超分子概念衍生而来, 建立 在可逆共价反应基础上的动态体系. 与超分子中的弱 分子间作用力相似, 可逆反应也具有自我修正与可适 应性，能随着环境变化或刺激源的加入而优化自身组 分状态 ${ }^{[1,2]}$. 如今, 随着动态共价键化学的不断发展, 不 仅其体系复杂性有所提高、篮选机制越加清晰，而且 也提供了将小分子网络作为整体进行研究的新视角, 在药物发现、生物结构的探索、化学催化、功能材料 等领域有多方面的应用 ${ }^{[3 \sim 6]}$.

酶是动态体系的重要刺激源. 作为生物体内化学 反应的天然催化剂, 酶催化因其专一性、高催化效率 和温和的反应条件而备受关注，在有机小分子合成乃
至聚合物的制备中都得到广泛应用. 尤其在医药中间 体的合成中，相对于其他有机或金属催化剂，酶催化 的高立体选择性和绿色化学特征显示出较多优势, 是 未来主要发展方向之一 ${ }^{[7]}$.

当动态共价键化学与酶催化相结合, 一方面, 可逆 反应有助于底物异构体消旋, 使得未被选择的异构体 在消旋后也经由酶催化动态动力学拆分, 可进一步提 高不对称有机合成的产率和对映体过量 $(\mathrm{ee})^{[8]}$; 另一方 面, 动态组合化学的响应性和高效篮选效率可为酶的 底物结构选择性提供重要信息, 特别是在同一体系相 同条件下的结构竞争性, 更具参考价值 ${ }^{[9]}$. 本文将主要 围绕以上两个方面的应用进行阐述(图1).

引用格式: 张燕, 陈敬华. 动态共价键化学在酶催化中的应用. 中国科学: 生命科学, 2019, 49: 605-614

Zhang Y, Chen J H. Dynamic covalent chemistry in enzyme catalysis (in Chinese). Sci Sin Vitae, 2019, 49: 605-614, doi: 10.1360/N052018-00245 


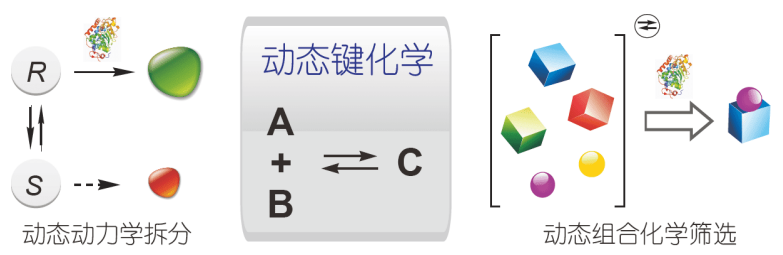

图 1 动态键化学与酶催化

Figure 1 Dynamic covalent chemistry in enzyme catalysis

\section{1 动态共价键化学简介}

\section{1 动态共价键化学的概念}

动态共价键化学是建立在可逆共价反应基础上的 新反应形式. 长久以来, 反应的可逆性被视为有机合成 中的一大弊端, 但如果利用这一特质, 不以单分子为研 究对象, 而采用针对超分子化学的处理方法, 将多反应 网络视为统一整体, 则衍生了动态共价键化学. 在动态 体系中，处于平衡状态的各组分可对外界刺激产生响 应，并相应地改变自身结构、组成形式以适应新环境. 因此, 取决于所加入刺激物的属性, 该类动态体系可用 于化学、生物信息的笁选和传递等多个领域 ${ }^{[10]}$.

理论上, 只要存在合适的催化剂以降低反应能垒, 大部分化学反应都有潜在可逆性. 但目前可用于动态 体系的可逆共价反应较有限, 主要分为五大类 ${ }^{[11]}$ : 亚 胺合成与交换反应; 二硫键反应; 基于碳氧、碳氮双 键的亲核加成反应; 烯的加成、复分解反应; 硼酸酯 的生成反应. 其中, 亚胺 ${ }^{[12]}$ 和二硫键 ${ }^{[13]}$ 的反应因为反 应迅速、可控性好, 用途最为广泛. 嗍酸酯的反应由 于生物相容性高，尤其在涉及糖的生物识别中有一定 的应用基础 ${ }^{[14]}$. 此外，基于碳氧、碳氮双键的亲核加 成反应因引入了新的手性中心, 在酶催化不对称合成 中有较高的应用潜力.

\section{2 动态共价键化学的特征}

动态体系的建立通常涵盖三个要素: 起始构建单 元、可逆共价反应和篮选压力. 构建单元与可逆共价 反应的选择需主要考虑两方面：共存性好，无不良副 反应的发生，不同反应类型之间没有干扰; 在丰富结 构多样性的同时，以具体应用为导向，不影响关键功 能基团与靶点的相互作用.

动态体系的快速可逆性是应用的关键，可直接影 响篮选效率. 尤其在多组分、多反应类型的体系中,
动态平衡点和平衡时间的确立显得异常重要. 判断方 法主要有两种 ${ }^{[11]}$ : 从原料和产物两端分别开始建立动 态系统，观察能否达到相同的平衡点并记录所需时间; 在动态体系上改变环境参数, 观察能否达到新的平衡 点及所需时间.

对于动态体系第三要素——篮选压力, 其表现形 式多样，可来自于外部，包括金属、离子、酶等外来 刺激的添加, 也可来自于体系内部的变化, 如结晶过 程等. 然而, 大多数篎选过程基于可逆的超分子识别 (图2A), 所诱导的聚集效应不明显, 不利于组分变化 的检测. 但如果动态体系连接了不可逆酶催化反应(图 $2 \mathrm{~B})$, 则重要组分的缺失会形成强大驱动力, 促使整个 体系向被选择的方向转化 ${ }^{[15,16]}$. 采用此策略, 一方面, 聚集放大效应明显，组分变化情况易于检测; 另一方 面, 也为酶催化的底物选择性提供了丰富信息.

\section{3 动态共价键化学与酶催化}

现有动态体系所涉及的不可逆转化以酶促反应为 主，也包括其他结晶 ${ }^{[17]}$ 、载体吸附 ${ }^{[18]}$ 等过程. 当动态 可逆反应与酶催化相结合时, 单反应体系可促进底物 $\mathrm{R}$ 和 $\mathrm{S}$ 的消旋，最直接的应用就是基于动态动力学拆分 的不对称合成. 此外, 生物酶的活性中心结构较复杂, 当用作有机合成催化剂之前, 对其底物选择性情况仍 需一一探索, 以获得最佳催化效果. 动态化学的多反 应、多组分、可适应性特征则可实现在同一体系中对 多种底物的同时比较, 显著提高篮选效率和准确性 ${ }^{[19]}$.

\section{2 动态化学在酶催化手性合成中的应用}

\section{1 动态可逆反应与酶催化动态动力学拆分}

酶催化动力学拆分是获得手性化合物的主要途径 之一 ${ }^{[20]}$. 然而, 该方法最大的弊端存在于: 即使酶对底 物异构体的选择性非常高, 催化反应的最大理论产率 只有 $50 \%$ (图3A), 因此很大程度上限制了产业化应用.

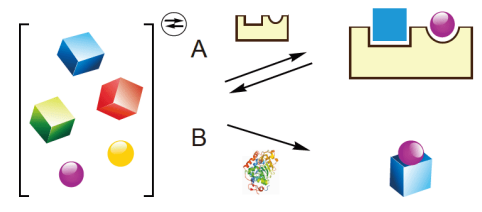

图 2 动态体系中的超分子识别(A)与不可逆酶催化(B) Figure 2 A: Supramolecular recognition and B: nonreversible enzyme catalysis within dynamic systems 
A

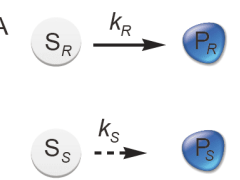

B

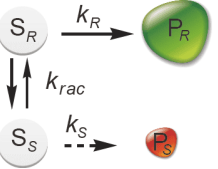

图 $3 \mathrm{~A}$ : 动力学拆分; $\mathrm{B}$ : 动态动力学拆分

Figure 3 A: Kinetic resolution. B: Dynamic kinetic resolution

动态动力学拆分概念的引入, 即在酶催化的同时促使 底物R和S异构体消旋，理论产率可提高到100\% (图 $3 \mathrm{~B})$ ，显著降低生产成本 ${ }^{[21]}$. 该策略中常用的消旋方法 为金属 ${ }^{[22]}$ 或异构酶 ${ }^{[23]}$ 介导，此外，可逆共价反应也可 起到相同的作用, 而且反应条件温和、操作简单 ${ }^{[24]}$.

在动态动力学拆分中最常用到的酶为脂肪酶. 一 方面因其化学、手性选择性高，并且催化机理、活性 中心结构都已研究得较透彻, 是不对称合成中的重要 生物催化剂 ${ }^{[24]}$; 另一方面，脂肪酶的来源丰富、价格 低廉, 在工业界也应用广泛 ${ }^{[25]}$. 此外, 其他生物酶, 包 括枯草杆菌蛋白酶 ${ }^{[26]}$ 、腈水解酶 ${ }^{[27]}$ 等, 在动态动力学 拆分中也有应用.

为了获得更高的产率和对映体过量值，该类基于 可逆反应的动态动力学拆分需满足 3 个条件 ${ }^{[28]}$ : 酶催 化反应的手性选择性较高; 可逆反应的速率大于慢反 应异构体的转化速率; 可逆反应与酶催化体系的条件 兼容. 目前，在动态动力学拆分中采用的可逆反应类 型以羰基、亚胺的亲核加成为主，不仅因为其反应条 件温和、可逆性质较高, 而且新手性中心的生成/破坏 可实现 $\mathrm{R}$ 与 $\mathrm{S}$ 异构体的转化, 为下一步酶催化高效不对 称合成提供底物. 下面就这几类常用可逆反应类型在 动态动力学拆分中的应用做简单介绍.

\section{2 基于羰基加成反应的动态动力学拆分}

羰基的亲核加成反应在有机合成中较常见，是传 统合成中受可逆性质困扰而影响产率的类型. 但在动 态体系中，其温和的可逆反应条件反而成为优势．根 据亲核试剂的不同，可逆羰基加成反应主要包括：半 缩醛、硫代半缩醛、半胺醛、氰醛、硝基羟醛反应等 类型(图4). 除了硝基羟醛反应需要碱的催化以形成 $\alpha$

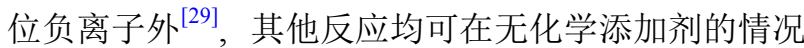
下快速进行, 因此是动态动力学拆分中较好的消旋 策略.

半缩醛反应是最早被开发的可逆共价反应之一,

$$
\text { 硫代半缩醛反应 }
$$

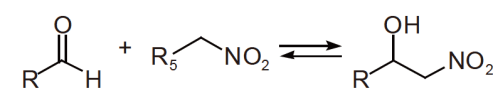

图 4 在动态动力学拆分中常用的羰基加成反应

Figure 4 The frequently used carbonyl addition reactions in dynamic kinetic resolution

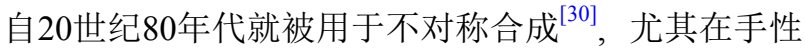
呋喃酮的制备中较为常见. 例如, Feringa和Kellogg课 题组 ${ }^{[31]}$ 将半缩酫加成反应与脂肪酶的不可逆乙酰化 结合，制备了一系列呋喃酮产物，其中化合物 3 在获得 $>99 \%$ ee的同时, 产率也达90\% (图5A). Zwanenburg课 题组 ${ }^{[32]}$ 在此基础上丰富了呋喃酮母核的取代基团 $(4$, 图5B), 并将骨架扩展到六元环的吡喃酮 $(5 \text {, 图5C) })^{[33]}$, 所得产率和ee值都较高.

与半缩醛相比, 半胺醛反应的可逆性需要较高的 温度才能进行，相应地，可得到多种手性吡咯酮化合 物(图6A) ${ }^{[34,35]}$. 另一方面, 硫代半缩醛反应因为颈基的 高亲核性，也能在常温条件下实现对羰基的快速加 成 ${ }^{[36]}$, 并连接酶不对称催化, 可获得 1,3 -氧硫环戊烷母 核 $(12,15$, 图6B和 $\mathrm{C})$, 而该类结构是合成抗艾滋病药物 拉米夫定(Lamivudine)的重要中间体 ${ }^{[26,37,38]}$.

氰基作为亲核试剂对羰基的亲核加成也比较直接, 可在常温、无添加剂的条件下进行. 而在碱的存在下, 反应速度有所加快. 考虑到氰化氢的毒性, 一般丙酮合 氧化氢(17)可作为氧基的替代供体, 并且与酶催化反应 显示出较好的兼容性, 经脂肪酶催化的乙酰化反应, 产 物的转化率和ee值都较高(图7A) ${ }^{[39,40]}$. 此外, 碱性条件 也是硝基羟醛的高效催化环境 ${ }^{[41]}$, 但为了避免产生第 二个难以控制的手性中心，常以对称结构的2-硝基丙 烷(21)作为亲核试剂. 同样，经脂肪酶催化的不对称酰 化反应, 产物异构体能得到有效分离(图7B) ${ }^{[42]}$. 


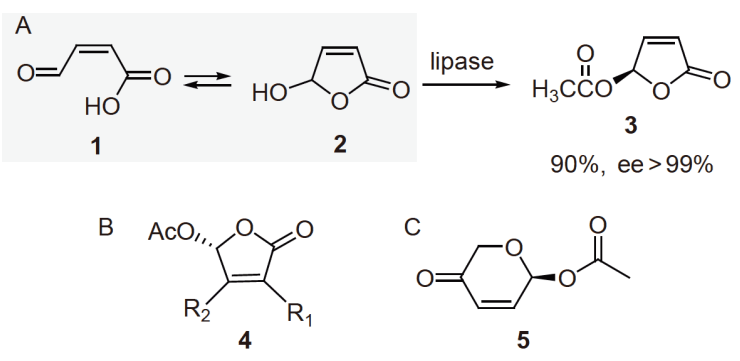

图 5 半缩醛反应在动态动力学拆分中的应用举例 ${ }^{[31 ~ 33]}$

Figure 5 Applications of hemiacetal formation reaction in dynamic kinetic resolution ${ }^{[31-33]}$
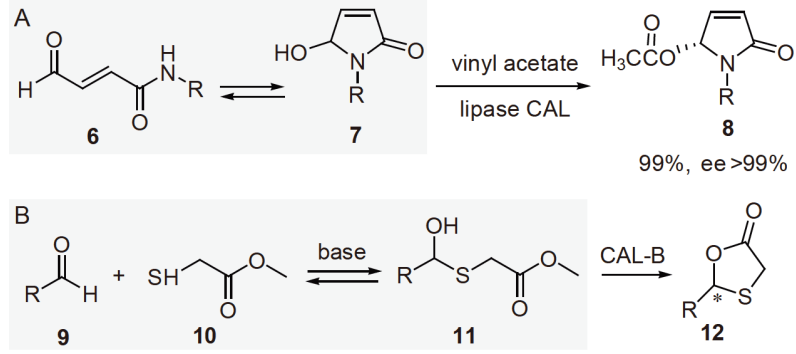

$79 \%$, ee $=90 \%$

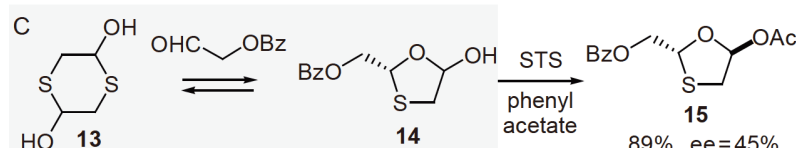

图 6 半胺醛反应 $(A)^{[35]}$ 及硫代半缩醛反应 $(B, C)^{[26,37]}$ 在动 态动力学拆分中的应用举例

Figure 6 Applications of $(A)^{[35]}$ hemiaminal and $(B, C)^{[26,37]}$ hemithioacetal formation reactions in dynamic kinetic resolution

$$
\begin{aligned}
& \text { A }
\end{aligned}
$$

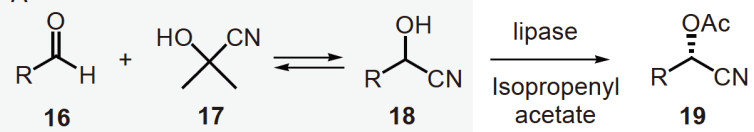

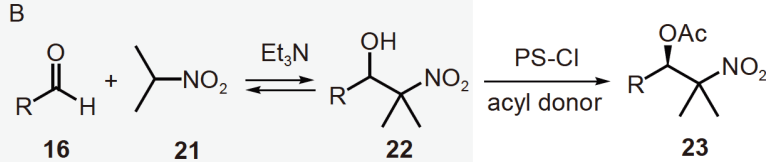

图 7 氰醛反应 $(\mathrm{A})^{[40]}$ 及硝基羟醛反应 $(\mathrm{B})^{[42]}$ 在动态动力学 拆分中的应用举例

Figure 7 Applications of $(\mathrm{A})^{[40]}$ cyanohydrin and $(\mathrm{B})^{[42]}$ nitroaldol reactions in dynamic kinetic resolution

\section{3 基于亚胺加成反应的动态动力学拆分}

与羰基相似，亚胺中的碳氮双键也能成为各亲核 试剂进攻的位点，并提供相似的可逆性质。在已有的 动态体系中，这些常用的亲核试剂包括羟基化物、統
基化物和氰基化物，相应地生成N,O-缩醛、N,S-缩醛 和Strecker反应产物(图8).

考虑到亚胺生成反应也可逆，能与该类亲核加成 反应串联，进一步提高动态体系的复杂度并简化反应 原料. Ramström课题组 ${ }^{[43]}$ 就建立了该类串联体系, 结 果显示, $\mathrm{ZnBr}_{2}$ 催化剂的加入可增强双重反应的可逆 性. 经脂肪酶介导的乙酰化反应，产物 26 以较高的产 率和 $90 \%$ 的ee值获得(图9A). Burk课题组 ${ }^{[27]}$ 也对该串 联反应进行了探索，发现1,8-二氮杂二环[5.4.0]十一 碳-7-烯(DUB)的加入或 $\mathrm{pH}>10$ 的强碱性条件也可提供 一定的异构体消旋速率, 经下一步腈水解酶催化, 获得 ee值较高的手性氨基酸产物(29, 图9B).

\section{3 动态组合化学在酶底物篮选中的应用}

当动态体系中的可逆反应建立在多组分、多反应 体系上, 则成为动态组合化学 ${ }^{[44,45]}$. 因该类体系的刺激 响应性、组分可适应性等特征，为即时、高效的生物 篮选提供了良好的平台. 与动态体系的其他应用相似，

$N, O$-缩醛反应

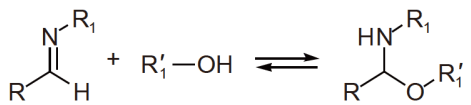

$N, S$ - 缩醛反应

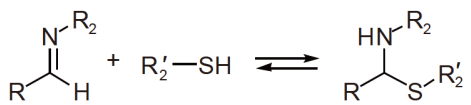

Strecker反应

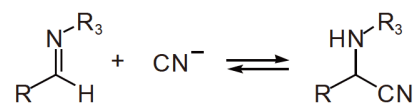

图 8 在动态动力学拆分中常用的亚胺加成反应

Figure 8 Applications of imine formation reaction in dynamic kinetic resolution
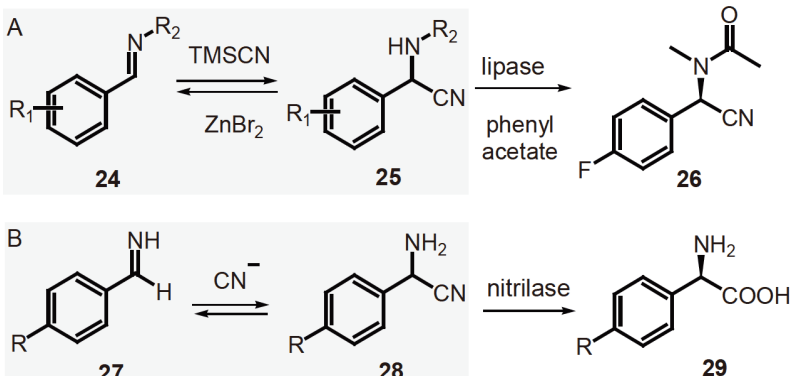

图 9 亚胺-Strecker反应在动态动力学拆分中的应用举 例 ${ }^{[27,43]}$

Figure 9 Applications of imine-Strecker reaction in dynamic kinetic resolution ${ }^{[27,43]}$ 
刺激源可来自于外部, 也可由体系内部产生. 当酶作为 篮选压力时, 动态组合库可用于酶催化底物的篮选, 并 且由于组分的多样性, 提供了较多的底物结构信息, 因 而针对酶的篮选放大效应也更为明显.

现有的动态组合化合物库的形式多样，复杂程度 不一，根据所含可逆共价反应的类型，可大致分为单 反应体系、双重串联/并联体系和复杂体系(图10).

\section{1 基于单反应的动态化合物库}

单反应体系因为组成简单、易于调控，是动态组 合化学中比较常见的类型. 硫酯交换反应是第一个将 动态体系与酶催化底物笁选相结合的例子 ${ }^{[46,47]}$. 将硫 代胆碱(31)与不同取代基团的硫酯(30)混合，经交换反 应得到可作为胆碱酯酶催化底物的新硫酯(32, 图11). 在加入胆碱酯酶进行水解反应后，结果显示，小基团 取代的底物更容易被水解，而稍大的支链基团则明显 减慢酶催化速度.

胆碱酯酶对底物的篮选主要限于化学结构上的差 异. 具有手性选择性的脂肪酶则可精确到底物的手性 位点, 为不对称合成提供更有效的信息. 至今, 基于多 种可逆反应的动态体系已被开发，包括硝基羟醛反 应、硫代半缩醛反应、氭醛反应等. 多种共价键连接 方式不仅丰富了底物的结构多样性，而且为其他应用 提供了篮选平台. 在加入脂肪酶进行底物篮选、催化 后, 都显示了较好的化学、区域、手性三重高选择性.

以基于硫代半缩醛反应的动态体系为例，将不同 结构的醛与链长不一的放基烷基酸甲酯混合, 经硫代 半缩醛反应得到两类中间体 40,41 供脂肪酶进行分子 内环酯化(图12) ${ }^{[48]}$. 结果显示, 在五元环和六元环两类 潜在产物中, 只有五元环被选择性合成, 并且醛上取代 基对反应的正面影响程度为: 短链脂肪基团 $>$ 长链脂 肪基团 $>$ 芳香基团. 最终, 异丙基取代的五元环产物 42 以较高的转化率和 $89 \%$ 的ee值从动态体系中被选择性 合成.

\section{2 基于双重反应的动态化合物库}

虽然动态共价反应大多条件温和，但将两个或两 个以上不同反应类型与酶催化置于同一体系，在条件 兼容的前提下避免其他可能的副反应，仍具有一定的 挑战性. 双重反应共存的表现形式主要包括串联和并 联两种.

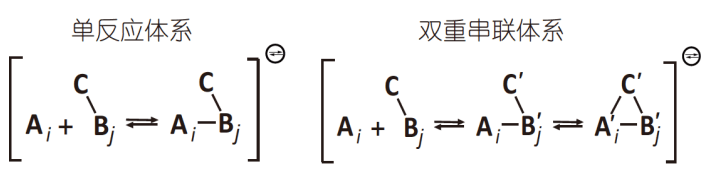

双重并联体系

复杂体系

$$
\left[\begin{array}{l}
\mathrm{A}_{i}+\mathrm{B}_{j} \rightleftarrows \mathrm{A}_{i} \mathrm{~B}_{j} \\
\mathrm{C}_{i}+\mathrm{D}_{j} \rightleftarrows \mathrm{C}_{i} \mathrm{D}_{j}
\end{array}\right]^{\ominus} \quad\left[\begin{array}{c}
\mathrm{A}_{2}-\mathrm{B}_{j}=\mathrm{A}_{i} \mathrm{~B}_{j} \\
\mathrm{~A}_{1}\|\|_{\mathrm{A}_{3}}-\mathrm{D}_{j}=\mathrm{C}_{i} \mathrm{D}_{j}
\end{array}\right]^{\ominus}
$$

图 10 动态组合化学体系中常见的可逆反应类型

Figure 10 Types of reversible reactions in dynamic combinatorial systems

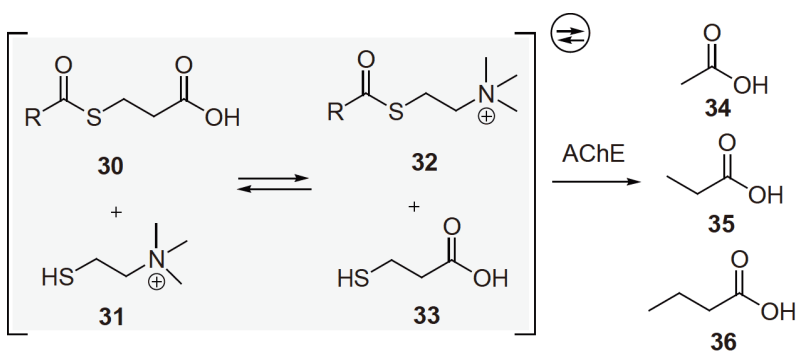

$\mathrm{R}=$ methyl-; ethyl-; propyl; butyl-; pentyl-

图 11 胆碱酯酶催化的单反应动态化合物库 ${ }^{[47]}$

Figure 11 Single dynamic system catalyzed by cholinesterase ${ }^{[47]}$

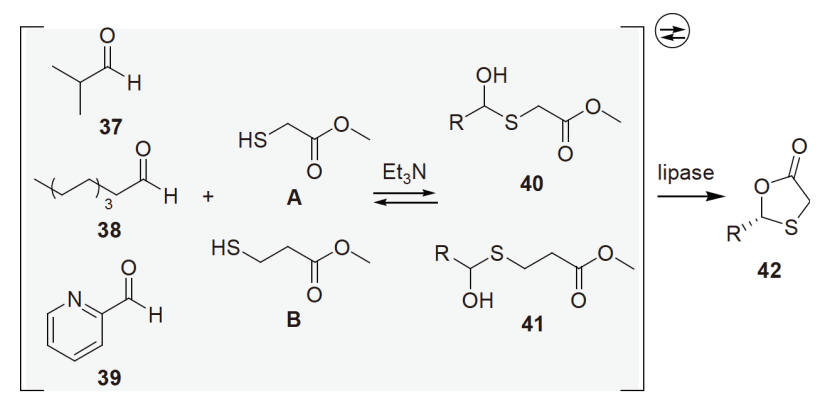

图 12 脂肪酶催化的单反应动态化合物库 ${ }^{[48]}$

Figure 12 Single dynamic system catalyzed by lipase ${ }^{[48]}$

串联反应在有机合成中比较常见，如果在某一条 件下同时可逆，则构成双重动态化合物库，进而可从 相对简单的原料获得结构复杂的酶催化底物. 现有的 该类动态体系主要涉及亚胺-Strecker反应 ${ }^{[43]}$ 和 Michael-Henry反应 ${ }^{[49]}$. 以后者为例, 从两个硝基苯乙烯 $(43,44)$ 原料出发, 经过Michael加成与分子内Henry环 合两步反应, 在共同催化剂强碱的存在下, 整个体系可 逆性良好, 所得两个中间体分别含有三个手性中心, 共 16 个异构体. 在加入脂肪酶进行不对称乙酰化后, 有且 
仅有一个异构体产物 48 被选择性合成(图13A).

如果串联反应为动态体系提供了相对复杂的结构 类型, 并联反应则能丰富动态体系的多样性. 较经典的 例子是硝基羟醛反应和硫代半缩醛反应在同一体系中 的共存 ${ }^{[00]}$. 在这一动态系统中, 醛作为共同的原料可 同时与2-硝基丙烷 $(\mathbf{C})$ 和1-丁硫醇反应 $(\mathbf{D})$, 得到两种结 构类型的六个中间体. 经脂肪酶催化的乙酰化, 只有 $3-$ 硝基取代的芳香醛被选择性转化，并且硝基羟醛反应 产物(52C)和硫代半缩醛反应产物(53D)之比为 $17: 1$, 产物ee值也均在 $90 \%$ 以上，显示出较高的反应类型、 化学结构和手性三重选择性(图13B).

\section{3 基于复杂反应体系的动态化合物库}

以进一步提高动态体系的复杂程度，多种类型的 可逆反应被置于同一体系. 与单一或双重反应的动态 体系相比，多个可逆反应的同时存在对组分的相容性 提出了更高要求.

Ramström课题组 ${ }^{[51]}$ 尝试将亚胺生成反应、亚胺
交换反应、硝基羟醛反应、硫代半缩醛反应以及 $\mathrm{N}, \mathrm{S}-$ 缩醛反应五种类型交错于同一体系. 酶催化过程也被 赋予多种可能性，乙酰化试剂的加入理论上可同时生 成链状乙酰化产物和环合产物(图14). 结果显示只有 两个N,S-缩醛中间体被转化成噻唑烷酮产物。

\section{4 展望}

作为生物靶点的模型, 酶催化在动态键化学中的 应用不仅丰富了动态体系的形式、诱导开发了新的可 逆反应类型, 而且为酶的催化属性、底物选择性提供 了较多信息, 为进一步针对疾病蛋白靶点的研究提供 了有力工具和理论基础. 在已有的动态体系中，单个 可逆反应可介导底物异构体消旋, 并通过动态动力学 拆分方法提高不对称催化的产率和ee值. 此外, 多组 分、多反应类型的动态化学体系可成为酶催化底物的 高效篮选平台, 以获得最优底物结构. 其他应用形式还 包括人工酶的设计 ${ }^{[52]}$ 、蛋白抑制剂的搜寻 ${ }^{[53]}$ 、酶的
A

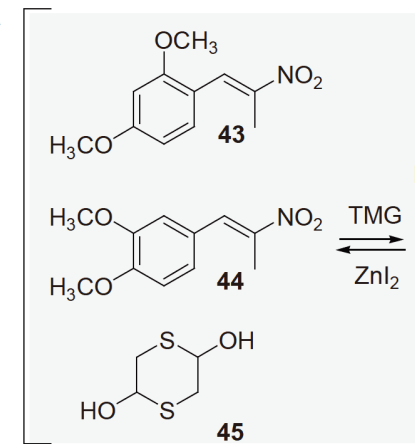<smiles>COc1ccc(C(SCC=O)C(C)[N+](=O)[O-])c(OC)c1</smiles><smiles>C=CCSC(c1ccc(OC)c(OC)c1)C(C)[N+](=O)[O-]</smiles><smiles>COc1ccc(C2SCC(O)C2(C)[N+](=O)[O-])c(OC)c1</smiles><smiles>COc1ccc([C@@H]2SCC(OC(C)=O)[C@@]2(C)[N+](=O)[O-])cc1OC</smiles>

$47^{\prime}$

B

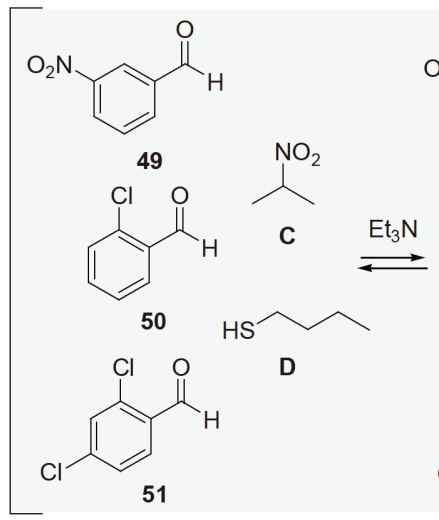<smiles>CC(C)(C)C(O)c1cccc([N+](=O)[O-])c1</smiles>

49C<smiles>CC(C)(C)C(O)c1ccccc1Cl</smiles>

$50 \mathrm{C}$<smiles>CC(C)(C)C(O)c1ccc(Cl)cc1Cl</smiles>

51C<smiles>CCCCSC(O)c1cccc([N+](=O)[O-])c1</smiles>

49D

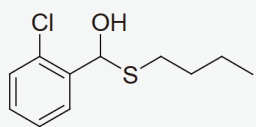

50D

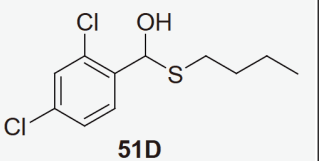

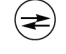

$97.7 \%$ ee

$\stackrel{\text { lipase }}{\longrightarrow} \quad 52 \mathrm{C}$

acyl donor<smiles>CCCCSC(OC(C)=O)c1cccc([N+](=O)[O-])c1</smiles>

图 13 脂肪酶催化的双重串联 $(\mathrm{A})^{[49]}$ 和并联 $(\mathrm{B})^{[50]}$ 动态化合物库

Figure 13 Double (A) cascade ${ }^{[49]}$ and (B) parallel dynamic ${ }^{[50]}$ systems catalyzed by lipase 


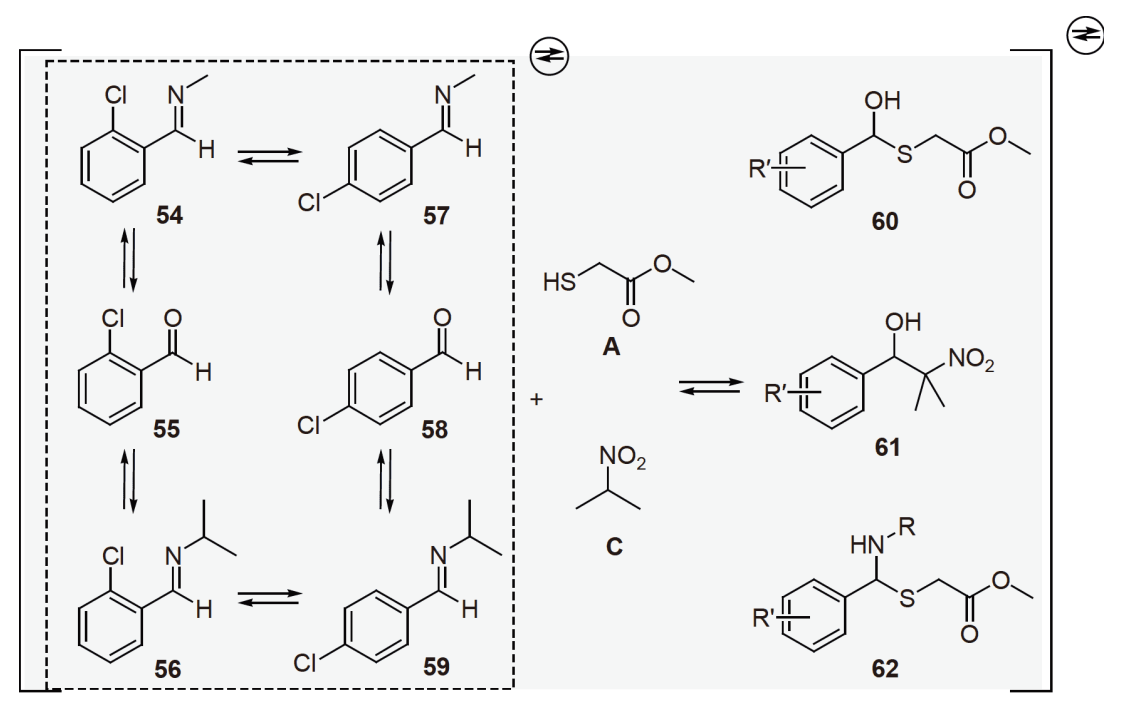

图 14 脂肪酶催化的复杂动态组合化学体系 ${ }^{[51]}$

Figure 14 Complex dynamic system catalyzed by lipase ${ }^{[51]}$

分类 ${ }^{[54]}$ 、动态键聚合物对酶的稳定与活化 ${ }^{[55,56]}$ 等, 为 动态键化学与酶催化之间搭建了新的桥梁. 虽然动态 共价键化学还处于起步发展阶段，但其在生物、材
料、合成等方面均表现了较高的潜力, 相信随着技术 的日趋成熟, 动态共价键化学在生物医药、材料领域 将表现出更广泛的实际应用价值.

\section{参考文献}

1 Lehn J M. From supramolecular chemistry towards constitutional dynamic chemistry and adaptive chemistry. Chem Soc Rev, 2007, 36: 151-160

2 Holub J, Vantomme G, Lehn J M. Training a constitutional dynamic network for effector recognition: storage, recall, and erasing of information. J Am Chem Soc, 2016, 138: 11783-11791

3 Rowan S J, Cantrill S J, Cousins G R L, et al. Dynamic covalent chemistry. Angew Chem Int Ed, 2010, 41: 898-952

4 Jin Y, Wang Q, Taynton P, et al. Dynamic covalent chemistry approaches toward macrocycles, molecular cages, and polymers. Acc Chem Res, 2014, 47: 1575-1586

5 Ramström O, Lehn J M. Drug discovery by dynamic combinatorial libraries. Nat Rev Drug Discov, 2002, 1: 26-36

6 Schaufelberger F, Ramström O. Kinetic self-sorting of dynamic covalent catalysts with systemic feedback regulation. J Am Chem Soc, 2016, 138: 7836-7839

7 Bornscheuer U T, Huisman G W, Kazlauskas R J, et al. Engineering the third wave of biocatalysis. Nature, 2012, 485: 185-194

8 Persson B A, Larsson A L E, Le Ray M, et al. Ruthenium- and enzyme-catalyzed dynamic kinetic resolution of secondary alcohols. J Am Chem Soc, 1999, 121: 1645-1650

9 Sakulsombat M, Zhang Y, Ramström O. Dynamic systemic resolution. In: Barboiu M, ed. Constitutional Dynamic Chemistry. Heidelberg: Springer, 2012. 55-86

10 Jin Y, Yu C, Denman R J, et al. Recent advances in dynamic covalent chemistry. Chem Soc Rev, 2013, 42: 6634-6654

11 Schaufelberger F, Timmer, B J J, Ramström O. Principles of dynamic covalent chemistry: principles, reactions, and applications. In: Zhang W, Jin Y, eds. Dynamic Covalent Chemistry. Hoboken: John Wiley \& Sons Ltd, 2017

12 Belowich M E, Stoddart J F. Dynamic imine chemistry. Chem Soc Rev, 2012, 41: 2003-2024

13 Otto S, Furlan R L E, Sanders J K M. Dynamic combinatorial libraries of macrocyclic disulfides in water. J Am Chem Soc, 2015, 122: 1206312064

14 Bapat A P, Roy D, Ray J G, et al. Dynamic-covalent macromolecular stars with boronic ester linkages. J Am Chem Soc, 2011, 133: 19832-19838 
15 Kulchat S, Chaur M N, Lehn J M. Kinetic selectivity and thermodynamic features of competitive imine formation in dynamic covalent chemistry. Chem Eur J, 2017, 23: 11108-11118

16 Ji Q, Lirag R C, Miljanić O S̆. Kinetically controlled phenomena in dynamic combinatorial libraries. Chem Soc Rev, 2014, 43: 1873-1884

17 Zhang Y, Legrand Y M, van der Lee A, et al. Ligand- and metal-driven selection of flexible adaptive dynamic host receptors. Eur J Org Chem, 2016, 2016: 1825-1828

18 Hsu C W, Miljanić O Š. Adsorption-driven self-sorting of dynamic imine libraries. Angew Chem Int Ed, 2015, 54: 2219-2222

19 Mondal M, Radeva N, Fanlo-Virgós H, et al. Fragment linking and optimization of inhibitors of the aspartic protease endothiapepsin: fragmentbased drug design facilitated by dynamic combinatorial chemistry. Angew Chem Int Ed, 2016, 55: 9422-9426

20 Keith J M, Larrow J F, Jacobsen E N. Practical considerations in kinetic resolution reactions. Adv Synth Catal, 2001, 343: 5-26

21 Verho O, Bäckvall J E. Chemoenzymatic dynamic kinetic resolution: a powerful tool for the preparation of enantiomerically pure alcohols and amines. J Am Chem Soc, 2015, 137: 3996-4009

22 Pellissier H. Organocatalyzed dynamic kinetic resolution. Adv Synth Catal, 2011, 353: 659-676

23 Turner N J. Enzyme catalysed deracemisation and dynamic kinetic resolution reactions. Curr Opin Chem Biol, 2004, 8: 114-119

24 Ghanem A, Aboul-Enein H Y. Lipase-mediated chiral resolution of racemates in organic solvents. Tetrahedron-Asymmetry, 2004, 15: 3331-3351

Ansorge-Schumacher M B, Thum O. Immobilised lipases in the cosmetics industry. Chem Soc Rev, 2013, 42: 6475-6490

Hu L, Schaufelberger F, Zhang Y, et al. Efficient asymmetric synthesis of lamivudine via enzymatic dynamic kinetic resolution. Chem Commun, 2013, 49: 10376-10378

27 Chaplin J A, Levin M D, Morgan B, et al. Chemoenzymatic approaches to the dynamic kinetic asymmetric synthesis of aromatic amino acids. Tetrahedron-Asymmetry, 2004, 15: 2793-2796

28 Huerta F F, Minidis A B E, Bäckvall J E. Racemisation in asymmetric synthesis. Dynamic kinetic resolution and related processes in enzyme and metal catalysis. Chem Soc Rev, 2001, 30: 321-331

29 Vongvilai P, Angelin M, Larsson R, et al. Dynamic combinatorial resolution: direct asymmetric lipase-mediated screening of a dynamic nitroaldol library. Angew Chem Int Ed, 2007, 46: 948-950

30 Smith J G, Fogg D E, Munday I J, et al. 1-Phenylisobenzofuran, 1-phenylnaphtho[2,3-c]furan, 1-phenylnaphtho[1,2-c]furan, and 3phenylnaphtho[1,2-c]furan via cyclic hemiaminal, hemiacetal, and acetal precursors. J Org Chem, 1988, 53: 2942-2953

31 van der Deen H, Cuiper A D, Hof R P, et al. Lipase-catalyzed second-order asymmetric transformations as resolution and synthesis strategies for chiral 5-(acyloxy)-2(5H)-furanone and pyrrolinone synthons. J Am Chem Soc, 1996, 118: 3801-3803

32 Thuring J W J F, Klunder A J H, Nefkens G H L, et al. Lipase catalyzed dynamic kinetic resolution of some 5-hydroxy-2(5H)-furanones. Tetrahedron Lett, 1996, 37: 4759-4760

33 van den Heuvel M, Cuiper A D, van der Deen H, et al. Optically active 6-acetyloxy-2H-pyran-3(6H)-one obtained by lipase catalyzed transesterification and esterification. Tetrahedron Lett, 1997, 38: 1655-1658

34 Cuiper A D, Kouwijzer M L C E, Grootenhuis P D J, et al. Kinetic resolutions and enantioselective transformations of 5-(acyloxy)pyrrolinones using candida antarctica lipase B: synthetic and structural aspects. J Org Chem, 1999, 64: 9529-9537

35 Sharfuddin M, Narumi A, Iwai Y, et al. Lipase-catalyzed dynamic kinetic resolution of hemiaminals. Tetrahedron-Asymmetry, 2003, 14: 15811585

36 Brand S, Jones M F, Rayner C M. The first examples of dynamic kinetic resolution by enantioselective acetylation of hemithioacetals: an efficient synthesis of homochiral $\alpha$-Acetoxysulfides. Tetrahedron Lett, 1995, 36: 8493-8496

37 Zhang Y, Schaufelberger F, Sakulsombat M, et al. Asymmetric synthesis of 1,3-oxathiolan-5-one derivatives through dynamic covalent kinetic resolution. Tetrahedron, 2014, 70: 3826-3831

$38 \mathrm{Hu}$ L, Ren Y, Ramström O. Chirality control in enzyme-catalyzed dynamic kinetic resolution of 1,3-oxathiolanes. J Org Chem, 2015, 80: 84788481

39 Inagaki M, Hiratake J, Nishioka T, et al. One-pot synthesis of optically active cyanohydrin acetates from aldehydes via lipase-catalyzed kinetic resolution coupled with in situ formation and racemization of cyanohydrins. J Org Chem, 1992, 57: 5643-5649

40 Effenberger F. Synthesis and reactions of optically active cyanohydrins. Angew Chem Int Ed Engl, 1994, 33: 1555-1564

41 Angelin M, Fischer A, Ramström O. Crystallization-induced secondary selection from a tandem driven dynamic combinatorial resolution process. J Org Chem, 2008, 73: 3593-3595 
42 Vongvilai P, Larsson R, Ramström O. Direct asymmetric dynamic kinetic resolution by combined lipase catalysis and nitroaldol (Henry) reaction. Adv Synth Catal, 2008, 350: 448-452

43 Vongvilai P, Ramström O. Dynamic asymmetric multicomponent resolution: lipase-mediated amidation of a double dynamic covalent system. J Am Chem Soc, 2009, 131: 14419-14425

44 Lehn J M. Dynamic combinatorial chemistry and virtual combinatorial libraries. Chem Eur J, 2015, 5: 2455-2463

45 Cougnon F B L, Sanders J K M. Evolution of dynamic combinatorial chemistry. Acc Chem Res, 2012, 45: 2211-2221

46 Larsson R, Pei Z, Ramström O. Catalytic self-screening of cholinesterase substrates from a dynamic combinatorial thioester library. Angew Chem Int Ed, 2004, 43: 3716-3718

47 Larsson R, Ramström O. Dynamic combinatorial thiolester libraries for efficientcatalytic self-screening of hydrolase substrates. Eur J Org Chem, 2006, 2006: 285-291

48 Sakulsombat M, Zhang Y, Ramström O. Dynamic asymmetric hemithioacetal transformation by lipase-catalyzed $\gamma$-lactonization: in situ tandem formation of 1,3-oxathiolan-5-one derivatives. Chem Eur J, 2012, 18: 6129-6132

49 Zhang Y, Vongvilai P, Sakulsombat M, et al. Asymmetric synthesis of substituted thiolanes through domino thia-michael-henry dynamic covalent systemic resolution using lipase catalysis. Adv Synth Catal, 2014, 356: 987-992

50 Zhang Y, Hu L, Ramström O. Double parallel dynamic resolution through lipase-catalyzed asymmetric transformation. Chem Commun, 2013, 49: $1805-1807$

51 Zhang Y, Ramström O. Thiazolidinones derived from dynamic systemic resolution of complex reversible-reaction networks. Chem Eur J, 2014, 20: $3288-3291$

52 Yoo S H, Lee B J, Kim H, et al. Artificial metalloprotease with active site comprising aldehyde group and Cu(II)cyclen complex. J Am Chem Soc, 2005, 127: 9593-9602

53 Mondal M, Hirsch A K H. Dynamic combinatorial chemistry: a tool to facilitate the identification of inhibitors for protein targets. Chem Soc Rev, 2015, 44: 2455-2488

54 Zhang Y, Jayawardena H S N, Yan M, et al. Enzyme classification using complex dynamic hemithioacetal systems. Chem Commun, 2016, 52: $5053-5056$

55 Zhang Y, Legrand Y M, Petit E, et al. Dynamic encapsulation and activation of carbonic anhydrase in multivalent dynameric host matrices. Chem Commun, 2016, 52: 4053-4055

56 Zhang Y, Feng W X, Legrand Y M, et al. Dynameric host frameworks for the activation of lipase through H-bond and interfacial encapsulation. Chem Commun, 2016, 52: 13768-13770 


\title{
Dynamic covalent chemistry in enzyme catalysis
}

\author{
ZHANG Yan $^{1,2}$ \& CHEN JingHua ${ }^{1,2}$ \\ 1 Key Laboratory of Carbohydrate Chemistry and Biotechnology, Ministry of Education, School of Bioengineering, Jiangnan University, \\ Wuxi 214122, China; \\ 2 School of Pharmaceutical Sciences, Jiangnan University, Wuxi 214122, China
}

Dynamic covalent chemistry is a new research area based on reversible covalent reactions. In such systems, the interconnected small molecules under dynamic equilibrium served as a responsive entirety, providing the possibility for constitutional selections and optimizations upon internal or external stimuli. When the stimulus is biocatalyst-enzyme, on one hand, the single reversible reaction enables fast racemization of substrates' enantiomers, leading to highly increased product's yield and enantiomeric excess (ee) through dynamic kinetic resolution; on the other hand, dynamic combinatorial chemistry can be used for screening of optimal enzyme substrates, with the increased versatility of the dynamic systems, the best fitted substrates can be efficiently obtained, to further achieve information on enzyme's catalytic activity and active sites, holding broad applications in future biomedical developments.

dynamic covalent chemistry, enzymatic catalysis, dynamic kinetic resolution, dynamic combinatorial chemistry

doi: $10.1360 / \mathrm{N} 052018-00245$ 